\title{
Substance Index for Volume 64
}

A54145D, E

$\mathrm{A} 54145 \mathrm{E}\left(\mathrm{Asn}_{3} \mathrm{Asp}_{9}\right)$

A-75943

A-90289 A, B

Abenquine A, B, C, D

20-nor-3 $\alpha$-Acetoxyabieta-5,7,9,11,13-pentaene

ACHN-490

Actinohivin

Actinohivin derivatives

Actinonin

Actinorhodin

Aflatoxin B1

AFN-1252

AH-135Y

Alkylguanidine, $N$-dodecyl- $N^{\prime \prime}$ -

Alkylguanidine, $N$-hexadecyl- $N^{\prime \prime}$ -

Alvespimycin

Amadacycline

Ambigol C

Ambiguine I isonitrile

Amikacin

3-Amino-5-hydroxybenzoic acid

Amoxicillin

Amphotericin

Amphotericin B

Ampicillin

Anishidiol

Ansamycin

Antimycin $A_{1 a}, A_{1 b}, A_{2 a}, A_{3 a}, A_{3 b}, A_{19}, A_{20}$

Antofloxacin

Apratoxin A

Aquastatin $\mathrm{C}$

Atacamycin A, B, C

Aurodox

Austamide

Avermectin

Avenolide

Azithromycin

Bafilomycin $\mathrm{C} 1$ amide

Bafilomycin C2 amide

Bafilomycin D, 29-hydroxy

Bafilomycin D, 9-hydroxy

Bafilomycin D, E, A1, B1, B2, C1, C2

Bafilomycin $\mathrm{K}$

BAL30072

Balofloxacin

BB83698

BC-3205, -3781, -7013

Bedaquiline

Benanomicin A, dexylosyl

Benanomicin B

Benzoxacystol

3-Benzyl-4-hydroxy-5-methyldihydrofuran-2-one Besifloxacin
79

111

163

495

763

401

413

551

551

413

97

9

413

163

469

469

635

413

401

401

625

35

625

89

469, 625, 735

625

211

35

661

413

401

213

775

197

45

89

781

$515,539,625$

159

159

159

159

159

391

413

413

413

413

413

133

133

453

267

413 $\begin{array}{ll}\text { Betulin derivatives } & 475\end{array}$

Biapenem 413

Bikaverin 9

Bireticulol $\quad 267$

Bisnoryangonin $\quad 349$

$\begin{array}{ll}\text { Borrelidin } & 51,381\end{array}$

Brevianamide A, F 45

Brevianamide E, deoxy $\quad 45$

Carbamido-cyclophanes 401

CB-182,325, -182,333, -182,348, -182,349, -182,350, 79

$-182,363,-182,390,-182,443,-182,597$

CB-183,315 413

Cefaclor $\quad 625$

Cefazolin $\quad 625$

Cefcapene $\quad 539$

Cefditoren 539

Ceftaroline fosamil $\quad 413$

Ceftazidime hydrate $\quad 625$

Ceftobiprole medocaril 413

Cematodin 401

Cethromycin $\quad 413$

CG400549 413

5-Chloro-2-(5-nitrofuran-2-yl)-1H-benzo[d] 667

imidazole

Ciprofloxacin

$73,321,539,625$

Ciprofloxacin hydrochloride $\quad 625$

$\begin{array}{ll}\text { Clarithromycin } & 539,625\end{array}$

Clarithromycin derivatives, 2,3-dehydro-3- 333

$O$-(3-aryl-E-prop-2-enyl)-10,11-anhydro

CNF-2024

635

Concanamycin A $\quad 89$

Coumermycin A2 133

Crotamine $\quad 327$

Cryptophycin-1, $-52 \quad 401$

Curacin A 401

CXA-101 413

Cyanopeptolin 963 A, 954

Cyanovirin-N 401

iso-Cycloheximide $\quad 163$

Cyclotryprostatin C $\quad 45$

Daidzein $\quad 133$

Dalbavancin $\quad 413$

Daptomycin $\quad 413$

Davallialactone $\quad 349$

Davallialactone, methyl $\quad 349$

Delafloxacin $\quad 413$

DHQ3, 7, 8

(E)-6-(2,4-Dihydroxyl-5-methylphenyl)-6-oxo-2- 645

hexenoic acid

6-(2,4-Dihydroxyl-5-methylphenyl)-6-oxohexanoic 645

acid

$\alpha$-Dimorphecolic acid 401

Dinapinone A, A1, A2 489, 503 
$N, N^{\prime}$-Diphenethylurea

Dolastatin 10, 15

Doramectin analogs

Doripenem

Doxorubicin

Dragomabin

Ebelactone A

Echinomycin

Elaiomycin B, C

Enoxacin

Ertapenem

Erythromycin

Erythromycin A 6,9-imino ether

Erythromycin A 6,9-imino ether 11,12-cyclic carbonate, 2-O-acetyl-4" $-O$-acylimidazolyl

Erythromycin A 6,9-imino ether 11,12-cyclic carbonate, $4^{\prime \prime}$-O-((2-cholorobenzyl)carbamoyl)

Erythromycin A 6,9-imino ether 11,12-cyclic carbonate, $4^{\prime \prime}$-O-((3-chlorobenzyl)carbamoyl)

Erythromycin A 6,9-imino ether 11,12-cyclic carbonate, $4^{\prime \prime}-O-((4-f l u o r o b e n z y l)$ carbamoyl $)$

Erythromycin A 6,9-imino ether 11,12-cyclic carbonate, $4^{\prime \prime}$-O-((4-methoxylbenzyl)carbamoyl)

Erythromycin A 6,9-imino ether 11,12-cyclic carbonate, $4^{\prime \prime}$-O-((benzyl)carbamoyl)

Erythromycin A 6,9-imino ether 11,12-cyclic carbonate, $4^{\prime \prime}$-O-((butyl)carbaomoyl)

Erythromycin A 6,9-imino ether 11,12-cyclic carbonate, $4^{\prime \prime}$-O-((isopropyl)carbamoyl)

Erythromycin A 6,9-imino ether 11,12-cyclic carbonate, $4^{\prime \prime}-O-(($ phenethyl $)$ carbamoyl $)$

Erythromycin A 6,9-imino ether 11,12-cyclic carbonate, $4^{\prime \prime}-O-(($ propenyl) carbamoyl)

Erythromycin A 6,9-imino ether, 2-O-acetyl

Erythromycin A 9-oxime

Erythromycin A, 10,11-didehydro-11-deoxy-3-Odescladinosyl-6-O-methyl-3-oxo-12-(1H-imidazole-1carboxylate)-2'-O-benzoyl

Erythromycin A, 10,11-didehydro-11-deoxy-3-O-descladinosyl-6-O-methyl-3-oxocyclic 1,12-carbonate-2'-Obenzoyl

Erythromycin A, 3-O-descladinosyl-6-O-methyl-2'-Obenzoyl

Erythromycin A, 3-O-descladinosyl-6-O-methyl-3oxo-cyclic 11,12-carbonate-2'-Obenzoyl

Erythromycin A, 3-O-descladinosyl-6-O-methyl-cyclic 11,12-carbonate-2'-O-benzoyl

Erythromycin D, 6-deoxy

Erythromycin D, alkynyl-6-deoxy

Erythronolide B, 6-deoxy

1-Ethylpropyloxycarbonyloxymethyl $(1 R, 5 S, 6 S)-2-$

[(3S,5S)-5-(N,N-dimethylcarbamoyl)-1-

(isobutyryloxymethyloxycarbonyl)pyrrolidin-3-ylthio]6-[(1R)-1-hydroxyethyl]-1-methylcarbapen-2-em-3carboxylate

\section{F-244}

Fasciculin A, B

483

19, 117
FD-594

Fidaxomicin

Finafloxacin $\quad 413$

Fleroxacin

5-Fluoro-2-(5-nitrofuran-2-yl)-1H-benzo[d]imidazole

$(2 S, 3 R)-\left(6^{\prime}-{ }^{2} \mathrm{H}\right)-N$-Formyl-3-hydroxy-Lkynurenine

Fosfomycin

FR179642

FR901379

Frenolicin B

Frenolicin $\mathrm{B}$ analogs

Fumitremorgin A, B, C

Fumitremorgin C, demethoxy

2-(4-(5-(Furan-2-yl)-2H-tetrazol-2-yl)butyl)isoindoline1,3-dione

4-(5-(Furan-2-yl)-2H-tetrazol-2-yl)butylamine

Furaquinocin D, I, J

Ganodermycin

Garenoxacin

Gatifloxacin

Geldanamycin

Geldanamycin derivatives

73, 539

Geldanamycin, 19-S-methyl

461, 519

177

519

Geldanamycin, 4,5-dihydro

Geldanamycin, 4,5-dihydro-19-S-methyl

461

519

Gemifloxacin

Genistein

Genistein, 7-O-methyl

Gentamicin

Germicidin A, B, C, D

Glucopiericidin C

Gramicidin $\mathrm{S}$ analogs

Granaticin

Grepafloxacin

GSK1322322

GSK2251052

Hapalindole T

(-)-Hirsutanol A, C

401

Hispidin

693

349

349

$\begin{array}{lr}\text { 8-Hydroxy-6,7-dimethoxy-3-methyl isocoumarin } & 267 \\ \text { 4-Hydroxy-3-nitrosobenzamide } & 93\end{array}$

13-Hydroxy-9Z,11E-octadeca di enoic acid 401

2'-(2-Hydroxyphenyl)-2,4'-bibenzoxazole-4-carboxylic 267 acid methyl ester

Hypholomine A, B

Hypothemycin

Iclaprim

ILX-651

Inonoblin $\mathrm{B}, \mathrm{C}$

Inoscavin A, B, C, D, methyl- 349

Inoscavin A, B, C, D, E 349

Interfungin A, B, C 349

Invictolide, 3,6,7-tri-epi- $\quad 385$

Isopenicillin N 447

Izuminoside A, B, C 271 
JBIR-101

JBIR-39, 40, 56, 57, 67, 68, 72, 73, 76, 77, 79, 80, 83, 84, 85,

689

$88,89,90,91,92,93,94,96,101,102,105,106,108$, $109,110,111,112,116$

JBIR-67, 73

JBIR-90, 91, 92, 93, 116

JNJ-32729463

Khatmiamycin

Landomycin X, Y, Z

Landomycinone, 11-deoxy

Lankamycin

Lankanamycin

Lasalocid

LBM-415

LC-KA05

Levofloxacin

Linezolid

Lobophorin E, F

Lotilibcin

Lovastain

Lyngbyastatin 4, 5, 6, 7

Maleic anhydride, 2,3-dimethyl-

Maleic anhydride, diphenyl-

Marinactinone A, B, C

ME1036

Megalomycin

Meridamycin

Meropenem analogs

Methyl 2-hydroxy-4-(2-hydroxy-3-methoxy-5-methylphenoxy)-6-methylbenzoate

Methyl 2-hydroxy-4-(3-hydroxy-5-methylphenoxy)-6methylbenzoate

7-Methyl-2-(5-nitrofuran-2-yl)-1H-benzo[d] imidazole

Methylenomycin

Methylinoscavin B, C, D

13-Methyltetradecanoic acid

Micafungin

Midecamycin

Milbemycin $\beta_{15}$

Minocycline

Minocycline hydrochloride

Mitomycin

Monacolin L, dihydro

Monapinone A, B, C, D, E

Monensin A phenylurethane sodium salt

Moxifloxacin

Mutactin

Mycotrienin II

N05WA963 A, B, D

Nemadectin

Nemonoxacin

Niddamycin

Niphimycin

281

367

413

655

$73,539,625$

667
Lankacidin C

Lankanolide, 8,15-dideoxy

12-Methyltetradecanoic acid
2-(5-Nitrofuran-2-yl)-1- $H$-benzo[d]imidazole

667

2-(5-Nitrofuran-2-yl)-3H-benzo[d]imidazole-4ol

667

Norfloxacin

Noscomin

Nostoflan

Notoamide A

NVC-422

Nystatin

NW-G01, G03

Okicenone

Oleandomycin

Oligomycin

Oligomycin A

Oligomycin A, 2,3-dihydro-

OPC-67683

Oritavancin

Oxisterigmatocystin A, B, C

Oxytetracycline

PA-824

Pahayokolide A

Pazufloxacin

Penicillin G

Pentalenic acid

Peplomycin

Perfloxacin

Phelligridimer A

Phelligridin A, B, C, D, E, F, G, H, I, J

Phellinin A1, A2

Phellinusfuran A, B

Phenalinolactone A, B, C, D

Phenatic acid A

Phenazine derivatives

Phenelfamycin G, H

2-(3-(5-Phenyl-2H-tetrazol-2-yl)propyl)isoindoline-1,3dione

2-(4-(5-Phenyl-2H-tetrazol-2-yl)butyl)isoindoline-1,3dione

3-(5-Phenyl-2H-tetrazol-2-yl)propylamine

4-(5-Phenyl-2H-tetrazol-2-yl)butylamine

Phthalide derivatives

Piericidin A

Pimaricin

Pinillidin

Piperacillin

73,625

401

401

45

413

89

789

459

89

89

155

155

413

413

193

19

413

401

413

539

65

309

73

349

349

349

349

229

163

729

257

571

571

571

571

723

267

89

349

Piperine

439, 673

427

Pivaloyloxymethyl $(1 R, 5 S, 6 S)-2-[(3 S, 5 S)-5-(N, N-$

dimethylcarbamoyl)-1-(isobutyryloxymethyloxycarbonyl)pyrrolidin-3-ylthio]-6-[(1R)-1-hydroxyethyl]-1methylcarbapen-2-em-3-carboxylate

Planktocyclin

401

Platencin

705

Platensimycin

705

Pleurone

587

413

PNU-100480

413

Prulifloxacin

183

Puberulic acid

729

2-(3-(5-(Pyridin-2-yl)-1H-tetrazol-1-yl)propyl)isoindo- 571 
2-(3-(5-(Pyridin-2-yl)-2H-tetrazol-2-yl)propyl)isoindoline-1,3-dione

2-(3-(5-(Pyridin-3-yl)-2H-tetrazol-2-yl)propyl)isoindoline-1,3-dione

2-(3-(5-(Pyridin-4-yl)-2H-tetrazol-2-yl)propyl)isoindoline-1,3-dione

2-(4-(5-(Pyridin-2-yl)-1H-tetrazol-1-yl)butyl)isoindoline1,3-dione

2-(4-(5-(Pyridin-2-yl)-2H-tetrazol-2-yl)butyl)isoindoline1,3-dione

2-(4-(5-(Pyridin-3-yl)-2H-tetrazol-2-yl)butyl)isoindoline1,3-dione

2-(4-(5-(Pyridin-4-yl)-2H-tetrazol-2-yl)butyl) isoindoline-1,3-dione

2-(5-(5-(Pyridin-2-yl)-1H-tetrazol-1-yl)pentyl)isoindoline-1,3-dione

2-(5-(5-(Pyridin-2-yl)-2H-tetrazol-2-yl)pentyl)isoindoline-1,3-dione

2-(5-(5-(Pyridin-3-yl)-2H-tetrazol-2-yl)pentyl)isoindoline-1,3-dione

2-(5-(5-(Pyridin-4-yl)-2H-tetrazol-2-yl)pentyl)isoindoline-1,3-dione

3-(5-(Pyridin-2-yl)-1H-tetrazol-1-yl)propylamine 3-(5-(Pyridin-2-yl)-2H-tetrazol-2-yl)propylamine 3-(5-(Pyridin-3-yl)-2H-tetrazol-2-yl)propylamine 3-(5-(Pyridin-4-yl)-2H-tetrazol-2-yl)propylamine 4-(5-(Pyridin-2-yl)-1H-tetrazol-1-yl)butylamine 4-(5-(Pyridin-2-yl)-2H-tetrazol-2-yl)butylamine 4-(5-(Pyridin-3-yl)-2H-tetrazol-2-yl)butylamine 4-(5-(Pyridin-4-yl)-2H-tetrazol-2-yl)butylamine 5-(5-(Pyridin-2-yl)-1H-tetrazol-1-yl)pentylamine 5-(5-(Pyridin-2-yl)-2H-tetrazol-2-yl)pentylamine 5-(5-(Pyridin-3-yl)-2H-tetrazol-2-yl)pentylamine 5-(5-(Pyridin-4-yl)-2H-tetrazol-2-yl)pentylamine Pyripyropene A

Quinaldic acid, 3-hydroxy

Quinoxaline-2-carboxylic acid

Radezolid

Radicicol

Ramoplanin $\mathrm{A}_{2}$

Rapamycin derivatives

Retapamulin

Retaspimycin

Reticulol

Rifampicin

Roflamycoin, 32,33-didehydro

Romidepsin

Scytovirin N

Secocycroheximide A, B

Seco-milbemycins E, F

Secopenicillide C

SF2768

Sitafloxacin

SMA76 a, b, c

SNX-2122

SNX-5422

Solithromycin
Somocystinamide A

401

Sparfloxacin

$89,539,625$

Spirotryprostatin A, B

45

Spirulan

401

Spoxazomicin A, B, C 303

SQ109 413

Squarrosidine $\quad 349$

Steffimycin 51

Sterigmatocystin, 5-methoxy 193

Streptolydigin $\quad 51$

Sulfoglycolipid 401

SW-163D $\quad 117$

Symplocamide A $\quad 401$

Tanespimycin $\quad 635$

Tautomycin $\quad 563$

Tebipenem pivoxil $\quad 413$

$\begin{array}{ll}\text { Teicoplanin } & 625\end{array}$

Telavancin 413

Telithromycin 413

Tetracenomycin F1, F2 9

2-(3-(5-(Thiophen-2-yl)-2H-tetrazol-2-yl)propyl)isoindo- 571 line-1,3-dione

2-(4-(5-(Thiophen-2-yl)-2H-tetrazol-2-yl)butyl)isoindo- 571 line-1,3-dione

3-(5-(Thiophen-2-yl)-2H-tetrazol-2-yl)propylamine 571

4-(5-(Thiophen-2-yl)-2H-tetrazol-2-yl)butylamine 571

Tigecycline 413

Tilmicosin, 10,11-dihydro-

$\begin{array}{ll}\text { Tobramycin } & 625,673\end{array}$

Tolyporphin 401

Torezolid phosphate $\quad 413$

Tosufloxacin $\quad 539$

TP-434 413

Triclosan 413

1-oxo-4(S),6,9-Trihydroxy-8-n-propyl-1,2,3,4-tetrahy- 459 droanthracene

Tryprostatins A, B $\quad 45$

$\begin{array}{lr}\text { Tylactone } & 89\end{array}$

$\begin{array}{ll}\text { TZT-1027 } & 401\end{array}$

Vancomycin $\quad 625$

Vancomycin derivatives $\quad 103$

Venturamide A, B 401

VER-52296NVP-AUY922 635

Verruculogen 45

Verticillin A $\quad 523$

Violacein $\quad 395$

Viridamide A 401

Viticolin A, B, C 183

WAP-8294A2, A1, A4, Ax13 373

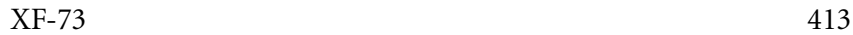

$\begin{array}{ll}\text { Xylaropyrone } & 217\end{array}$

$\begin{array}{ll}\text { YWA1 } & 9\end{array}$

Zabofloxacin $\quad 413$

Zearalenone $\quad 9$ 